\title{
CAUSAS DE LA SATISFACCIÓN LABORAL EN UNA ORGANIZACIÓN COMERCIALIZADORA MAYORISTA*
}

\author{
PEDRO MANUEL ZAYAS AGÜERO**, RAFAEL ANTONIO BÁEZ SANTANA***, JOSUÉ ZAYAS FERIA ${ }^{* * * * *}$ \\ \& MARIANELA HERNÁNDEZ LOBAINA******* \\ UNIVERSIDAD DE HOLGUÍN (CUBA)
}

Recibido/ Received/Recebido: 17/09/14 - Aceptado/ Accepted / Aprovado: 18/06/15

\begin{abstract}
Resumen
Este estudio tiene como objetivo determinar las causas que influyen en la satisfacción laboral en una comercializadora mayorista, permitiendo establecer las estrategias y las acciones para lograr un proceso de mejora continua de la organización. En la metodología se emplearon técnicas como: escalas, encuestas, entrevistas y observación directa, todas interrelacionadas, procediéndose a la triangulación de los resultados. Se validaron por expertos los instrumentos diseñados. El procesamiento estadístico se hizo usando el software estadístico SPSS. En los resultados de la investigación se detectaron los aspectos que inhibieron la satisfacción laboral en los empleados, así como el conjunto predominante de dimensiones e indicadores que identifican las principales insuficiencias existentes. Palabras clave: Satisfacción laboral; Motivación; Valoración; Percepción; Actitudes.
\end{abstract}

\section{CAUSES OF JOB SATISFACTION IN A MARKETING ORGANIZATION WHOLESALE}

\begin{abstract}
This study aims to determine the factors that influence job satisfaction in a wholesale marketer, allowing to establish strategies and actions to achieve a process of continuous improvement of the organization. The methodology used techniques such as: scales, surveys, interviews and direct observation, all interrelated and proceeding to the triangulation of results. It was validated by experts the designed instruments. The statistical processing was done using the SPSS statistical software. The results of the research aspects that inhibited job satisfaction among employees as well as the predominant set of dimensions and indicators that identify the major existent inadequacies were detected. Keywords: Job satisfaction; motivation; Assessment; Perception; Positions.
\end{abstract}

\footnotetext{
* Artículo resultado de investigación científica.

** Profesor de la Universidad de Holguín. Doctor en Ciencias Psicológicas. Dirección postal: Aniversario s/n Piedra Blanca Holguín (Cuba). CP 80100. Teléfono: (53 24) 480981. Correo electrónico: pedro@ucm.hlg.sld.cu.

**** Ingeniero Industrial y Master en Gestión de Recursos Humanos.

***** Licenciado en Economía.

******* Ingeniero Industrial.
} 


\title{
CAUSAS DA SATISFAÇÃO LABORAL EM UMA ORGANIZAÇÃO COMERCIALIZADORA ATACADISTA
}

\begin{abstract}
Resumo
Este estudo tem como objetivo determinar as causas que influenciam a satisfação laboral em uma comercializadora atacadista, permitindo estabelecer as estratégias e as ações para conseguir um processo de melhora contínua da organização. Na metodologia empregaram-se técnicas como: escalas, pesquisas de opinião, entrevistas e observação direta, todas inter-relacionadas, procedendo à triangulação dos resultados. Os instrumentos desenhados foram validados por peritos. O processamento estatístico fez-se usando o software estatístico SPSS. Nos resultados da pesquisa detectaram-se os aspectos que inibiram a satisfação laboral dos empregados, assim como o conjunto predominante de dimensões e indicadores que identificam as principais insuficiências existentes.

Palavras chave: Satisfação laboral; Motivação; Valoração; Percepção; Atitudes.
\end{abstract}

Zayas, P., Báez, R., Zayas, J. \& Hernández, M. (2015). Causas de la satisfacción laboral en una organización comercializadora mayorista. En: Revista de la Facultad de Ciencias Económicas de la Universidad Militar Nueva Granada. rev.fac.cienc.econ, XXIII (2), DOI: http://dx.doi.org/10.18359/rfce.1606.

JEL: J01, J21, J81.

\section{Introducción}

La gestión empresarial es objeto de estudio y centro de atención de representantes de diferentes ramas del conocimiento científico, empresarios y hombres de estado, por la incidencia de la misma en el desempeño organizacional y sus implicaciones en la esfera económica y consecuentemente en otras esferas de la vida social (Zayas, 1996, p. 7).

En el mundo actual, globalizado, cambiante, caracterizado por avances científicos y una poderosa tecnología, el hombre desempeña el papel fundamental para la organizacion, su funcionamiento $y$ su evolución.

La gestión del capital humano coloca el desarrollo humano como una prioridad dentro de las organizaciones, dejando de ser visto como un costo para ser considerado una inversión. Así se habla hoy día de capital humano, capital intelectual, potencial humano, talento humano, todos referidos al papel que tiene el hombre en la organización.

A la gestión del capital humano le corresponde impulsar los cambios organizacionales, y debe de tener un carácter holístico, sinérgico, proactivo y relacio- nal, por tanto, debe hacerse énfasis en la integridad y organicidad de cada subsistema y las interrelaciones intra e intersistemas.

Dentro de la gestión empresarial, el capital humano puede considerarse el más dinámico y es un producto de la interrelación entre las personas con la organización, las políticas, las normativas y los métodos que deben estar alineadas con la misión, la visión y los objetivos, aclarar los valores y principios de la organización, y ser capaces de movilizar a sus miembros en el propósito de impulsar los cambios y generar innovaciones.

Una correcta gestión del capital humano es fundamental para las organizaciones, porque esta significa un mayor grado de eficiencia, efectividad, eficacia y comprometimiento por parte de los trabajadores y por ende mayor productividad.

El estudio de la satisfacción laboral, es una problemática que surge desde los mismos procesos de división del trabajo y se sistematiza al surgir la psicología y la administración como ciencias. Es un problema complejo y controvertido desde el punto de vista científico, pues existen múltiples tendencias en el estudio tanto del hombre como de la actividad laboral. 
La satisfacción laboral tiene sus antecedentes en el psicólogo alemán Munsterberg (1913) que introdujo el tema con el libro "Psichology and industrial efficiency", pero el interés por el estudio surge al publicarse "Job Satisfacction" de Hoppock (1935).

La satisfacción laboral ha sido una de las variables más estudiadas en el ámbito de la actividad laboral, pero no existe un consenso en cuanto a su definición, incluso algunos especialistas, señalan que este concepto está libre de teoría, así vemos que en la actualidad no existe una definición unánime del concepto de satisfacción laboral.

Los especialistas de las ciencias sociales tienen distintas posiciones filosóficas, enfoques, escuelas, concepciones. Existen técnicas para elaborar conceptos pero va más allá, unos reflejan primordialmente las fuentes, los contenidos, las acciones y los resultados según en lo que los teóricos quieran hacer énfasis.

Todos los enfoques del trabajo desde Taylor (1911); Mayo (1933); Maslow (1954); Herzberg et al. (1959); McGregor (1960); Vroom (1964); Locke (1976); Hackman \& Oldham, (1980); Robbins \& Coulter (1996) hasta hoy, tienen una teoría sobre la motivación que se basa en la satisfacción de las necesidades y que es la base de la satisfacción laboral. Los trabajos de Herzberg son muy conocidos, entre el estudio de los factores que determinan la motivación por el trabajo, donde se realiza una distinción entre los factores de higiene y los motivadores, en esta investigación no se acepta esa clasificación, porque los fenómenos psicológicos pueden tener cualquier la estructura de las necesidades. La satisfacción laboral es un continuo, que va desde de la satisfacción hasta la insatisfacción y no un fenómeno separado.

Toda actividad de una persona está motivada, impulsada, dirigida, y movilizada, desde el punto de vista psicológico, por los motivos conscientes o inconscientes. Los motivos son los mecanismos psicológicos que impulsan y dan dirección a la actuación.

Según Robbins (2000, 2006), la motivación es la disposición a hacer algo, y que está condicionada por la capacidad de estos actos para satisfacer alguna necesidad. La motivación y la satisfacción laboral son formaciones distintas. La motivación, es eje de la satisfacción laboral. La motivación se refiere a los movimientos o impulsos para satisfacer un deseo o meta. La satisfacción laboral se refiere a la valoración de un objeto, proceso o fenómeno cuando se experimenta, o se vivencia algo, y la satisfacción cuando culmina una meta. En otras palabras, motivación implica el impulso hacia un resultado, mientras que la satisfacción laboral implica los resultados ya experimentados. Un trabajador puede estar motivado con el trabajo pero no satisfecho, y viceversa.

La motivación es una formación psicológica y es un elemento importante para lograr que se realicen los máximos esfuerzos en el cumplimiento de las metas de la organización, si no se toman en consideración las condiciones laborales donde se desempeña el trabajo.

Existen varias vertientes de la satisfacción laboral ${ }^{1}$ pero vamos a diferenciarlas en dos, los que dicen que la satisfacción laboral es una actitud y los que la ven como una percepción o otra formación psicologica. Aunque en el concepto pueden coexistir porque no son diferentes o contrarios, lexicamente, pero si se refieren a cosas diferentes, epistemologicamente y filosoficamente.

Los órganos de los sentidos son los que ponen a los sujetos en contacto con los objetos, entonces los fenómenos psíquicos empiezan por ahí desde los procesos, las formaciones, la personalidad hasta los factores sociales. La satisfacción laboral se puede valorar en objeto como el reflejo subjetivo-objetivo-subjetivo de lo cognitivo y afectivo, por eso es el efecto que uno percibe cognitivamente y afectivamente $y$ en virtud de esto se pueden adoptar actitudes positivas y negativas hacia el trabajo.

Existen varios procesos, fenómenos y formaciones complejas de la actividad laboral en una organización que no se pueden confundir, y son la percepción, la motivación, las actitudes y la valoración. Tubán (2000) plantea que existen también diferen-

Existen numerosas definiciones sobre la satisfacción laboral como la de Davis \& Newstrom (1999); Morse (1953); Larrainzar et al (2001); Locke (1976); Porter \& Lawler (1988); Robbins (2000); Spector (2002); Strauss \& Sayles (1992) pero no existe una definición unánime del concepto. 
cias entre la satisfacción laboral y las actitudes y dice: "Existe una gran confusión en el empleo del término "satisfacción en el trabajo" y los factores que contribuyen a ella. La revisión de los numerosos estudios efectuados en este campo nos lleva a la conclusión de que la satisfacción en el trabajo es casi cualquier cosa que un investigador mide cuando cree estar midiendo la "satisfacción en el trabajo". Añade "Aunque, en muchos casos, se utilizan como sinónimos, no lo son. Una "actitud" no es "satisfacción en el trabajo", aunque puede contribuir a ella (...) Una "actitud" de un empleado puede considerarse como la disposición para actuar de un modo más que de otro, en relación con factores específicos relacionados con el puesto" (Tubán, 2000, p. 1).

Padua (1979 citado en Hernández at al., 2006, p. 255) ejemplarmente plantea: "las mediciones de actitudes deben interpretarse como "síntomas" y no como "hechos" (...) La actitud es como una "semilla", que bajo ciertas condiciones puede "germinar en comportamiento". Las actitudes son como las disposiciones para actuar de una persona o un grupo en relación a los objetos, los fenómenos y las personas, son disposiciones de ánimo.

Las actitudes son una formación psicológica predominantemente de carácter afectivo, que se integra con la esfera cognitiva y conductual. Es como una expectativa, que bajo determinadas condiciones, puede hacerse realidad.

Desde el punto de vista epistemológico, se puede valorar el objeto como el reflejo subjetivo-objetivosubjetivo de lo cognitivo y afectivo, por eso la satisfacción laboral es el efecto que uno percibe cognitiva $y$ afectivamente y se puede adoptar actitudes positivas y negativas hacia el trabajo.

Este estudio tiene como una de sus finalidades valorar como los trabajadores perciben su trabajo, sus características, las condiciones y sus relaciones para tomar medidas que repercutan positivamente en la organización.

La percepción es el proceso que pone en contacto de forma directa e inmediata al sujeto con el medio y determina la forma de ver el mundo que tiene cada persona. Consiste en el reflejo y transformación mediante el cual los sujetos organizan e interpretan sus impresiones sensoriales con el fin de dar significado a su entorno. La percepción es objetiva, subjetiva, integral, selectiva y está en correspondencia con las motivaciones, intereses y experiencias de cada cual.

La valoración es una formación psicológica predominante cognitiva integrada a la esfera afectiva y es imagen subjetiva de una realidad objetiva. La percepción y la valoración es el eje de la satisfacción laboral.

En esta investigación se escoge el concepto de Zayas et al. (2014, p. 3) que plantea: "La satisfacción del trabajo es el resultado de la valoración cognoscitiva y afectiva, de lo que uno percibe de las características del trabajo y el reflejo de las características del trabajo en su medio".

Entre los factores socio-psicologicos más importantes está la satisfaccion del trabajo ha de estar entre los principales objetivos del trabajo de una organización. La importancia y la necesidad del estudio de la satisfacción laboral, radica en analizar los factores hombre- trabajo interrelacionados para el desarrollo de la actividad laboral, lo que permite una realización más eficiente de la misma, e incide en la competitividad de la organización; esto tiene una influencia en la disciplina, ausencias, llegadas tardes, rotación de personal, ineficiencia, relaciones interpersonales y otras.

La satisfacción laboral no es importante solo desde el punto de vista económico, sino también desde el punto de vista social, ya que los beneficios derivados de la misma impactan en la sociedad, pues esta sale del marco laboral del empleado y abarca el entorno de su vida.

Los directivos tienen que conocer las características generales y específicas de sus empleados y las causas que influyen en la satisfacción laboral, para así formular las políticas, estrategias y accionesque les permita actuar en función del mejoramiento estable de la organización.

Las tendencias más actuales en el campo empresarial tienen que estar basada precisamente en la sa- 
tisfacción laboral que experimentan los empleados en las organizaciones. En tal sentido la organización debe conocer las necesidades que experimentan sus trabajadores y crear las vías necesarias para su satisfacción. Eso constituye el núcleo principal de su motivación en el trabajo y el interés parte del hecho de que la motivación es la fuerza que mueve a las personas a realizar una acción o actividad humana.

\subsection{Dimensiones esenciales}

Brayfield \& Rothe (1951); Smith, Kendall \& Hulin (1969); Hackman \& Lawler (1971); Cuesta (1990); Peiró (1991); López (1994); Álvarez (2005), han contribuido a la construcción de las dimensiones para el estudio de la satisfacción laboral, no obstante las dimensiones a utilizar para dichos estudios están en las características de la organización que se investigue.

Para este trabajo se emplearon las dimensiones esenciales de la satisfacción laboral propuestas por Álvarez (2005, p. 19): la naturaleza y contenido de trabajo, trabajo en grupo y a su dirección, el esfuerzo y resultados individuales, condiciones de trabajo y condiciones de bienestar. Zayas et al. (2014) une a las dimensiones planteadas por Álvarez (2005) dos dimensiones: las normas, valores y costumbres y la estructura y otras variables de los aspectos socio-psicológicos.

Las dimensiones esenciales de la satisfacción laboral en este estudio son:

- La estructura: es la manera en que las acciones de las organizaciones se dividen, organizan y coordinan. Incluye una serie de aspectos como el control administrativo, niveles jerárquicos, división de funciones y tareas, relación e interdependencia entre los niveles que la forman, cadenas de mando, entre otros.

- La naturaleza y contenido de trabajo: se percibe el contenido de trabajo como variedad de habilidades, independencia, significación de la tarea, importancia, definición del contenido de trabajo, autonomía, organización y creatividad.

- Las normativas, valores y costumbres: es el conjunto de suposiciones, creencias, cultura, valores y normas que comparten sus miembros; crea el ambiente humano en que los empelados realizan su trabajo. En la cultura influye todo lo que sucede en la organización y crea el sello de la misma. Los indicadores de la cultura son: patrones de comunicación, sistemas y procedimientos, declaraciones de filosofías, historias y metas.

- El salario y la estimulación: dentro de esta se hayan la remuneración y compensación que está asociado a sistemas de pagos, promoción, posibilidades de superación y la evaluación del desempeño. Se juzga la estimulación por el reconocimiento, la suficiencia, la correspondencia, la justeza, el equilibrio entre los estímulos morales y materiales, organización y percepción del sistema de estimulación.

- Las condiciones de trabajo: se percibe la existencia de los materiales y medios necesarios para realizar el trabajo, la higiene y orden. Las condiciones ergonómicas son: las higiénicas, seguras y estéticas.

- Las condiciones de bienestar: el desarrollo personal y profesional, el horario de trabajo, el transporte, la alimentación, los servicios de salud, actividades recreativas, culturales y deportivas.

- Dimensiones esenciales vinculadas a aspectos socio-psicológicos:

- Las relaciones interpersonales y comunicación: Es el proceso de interacción social en el que intervienen al menos dos o más personas para intercambiar ideas, sentimientos, juicios, información, opiniones o instrucciones de trabajo. Las relaciones entre las personas, las relaciones personas-directivos, las relaciones entre los directivos, las posibilidades de interacción social, la preocupación por los problemas de las personas, la solución de conflictos, la pertenencia y la orientación y ayuda.

- Liderazgo y toma de decisiones: Es la influencia mediante la cual un individuo o 
grupo de persona pueden lograr que los miembros de una organización colaboren voluntariamente y con entusiasmo en el logro de los objetivos propuestos. El liderazgo es el proceso de influencia, estimulación y ayuda para transformar el potencial que posee un grupo en realidad. La escucha y la participación, la satisfacción con los métodos y el estilo de toma de decisiones, forman parte del liderazgo. Es la selección consciente, por parte de los directivos, de varias acciones entre alternativas posibles para producir un resultado esperado o deseado y cuyas consecuencias lo encadenan con el futuro. La toma de decisiones está asociada al liderazgo, la comunicación, la participación y al trabajo en equipo.

- Compromiso con el trabajo: Puede definirse como el grado en el cual una persona se identifica con su trabajo, participa activamente en él y considera su desempeño importante para la valoración propia (Zayas et al., 2014).

\subsection{Justificación}

En la comercializadora mayorista se realizó un diagnostico a los trabajadores en el año 2014, donde se detectaron deficiencias que ponen en riesgo la eficiencia de la organización. Las mismas se relacionan a continuación: problemas de la disciplina laboral, alta fluctuación, poca profesionalidad en el desempeño de la actividad fundamental.

Dado lo anteriormente expuesto con la situación problemática se define el problema científico siguiente: ¿Cuáles son las causas que influyen en la satisfaccion laboral en la Comercializadora Mayorista y qué estrategias y acciones implementar?

El objetivo general de la investigación es: Determinar las causas que influyen en la satisfaccion laboral en la organización para diseñar estrategias que logren la estabilidad laboral.

Para darle cumplimiento al objetivo general planteado se definieron los objetivos específicos: determinar el estado actual la satisfaccion laboral en la organización; analizar algunas variables que inciden en las áreas de los Almacenes y Seguridad y protección física en la organización; establecer las estrategias y las acciones pertinentes para lograr los niveles deseados de satisfacción laboral.

Para dar solución al problema científico planteado anteriormente se formula la siguiente hipótesis de investigación: existen la mayoría de los trabajadores de la comercializadora mayorista al responder a la escala de la satisfacción laboral, valoran las dimensiones, indicadores y los ítems expresando con insatisfacciones en su trabajo.

\section{Metodología}

En el desarrollo de la investigación se utilizaron los métodos teóricos y empíricos, tanto cuantitativos como cualitativos.

Entre los métodos teóricos empleados estuvieron: análisis y síntesis, histórico-lógico, inductivo-deductivo y sistémico- estructural, y los métodos empíricos como la observación y la experimentación. Dentro las técnicas de investigación que se utilizaron: las escalas, encuestas, entrevistas, entre otros.

\subsection{Población y muestra}

La muestra abarcó 88 trabajadores del área de Almacenes y el área de Protección física. Se proyectó investigar al $100 \%$ de la plantilla cubierta que abarcaba 92 trabajadores, procesándose 88 lo que representan el 96,5\%.

Del total de los trabajadores investigados, 83 son hombres para un $94,32 \%$ y 5 mujeres para tan solo un 5,68\%. De los trabajadores estudiados, 48 tienen entre 21 y 35 años de edad, representando un $54,55 \%$ del total. Le siguen en orden descendente los encuestados que tienen más de 45 años de edad, que son 20 para un $22,73 \%$, además que 19 encuestados se encuentran en el rango de edades ubicado entre los 36 y 44 años lo que representa el 21,59\%.

Con relación a la antigüedad, 36 trabajadores, tienen menos de 4 años en el centro para un 40,91\%; 29 
encuestados llevan en el centro entre 5 y 7 años para un 32,95\%; 14 han permanecido de 8 a 10 años para un $15,91 \%$ y con más de 10 años en el centro se ubican 9 de los encuestados para un $10,23 \%$.

De acuerdo al nivel escolaridad, 56 trabajadores tienen el nivel medio superior para un 63,64\%. El nivel superior es alcanzado por 19 , representando un $21,59 \%$ del total de encuestados y de nivel medio hay 13 encuestados para un $14,77 \%$.

\subsection{Diseño de los instrumentos y técnicas para la medición de la satisfacción laboral}

La construcción y evaluación cualitativa de la escala y los ítems, es parte de la relación teoría-praxis.
En esta tarea se utilizó el método del Rombo de las investigaciones, el que consiste en el eslabonamiento de las distintas etapas, tareas y métodos del proceso investigativo. En la modelación se parte de un punto, la definición del problema y los objetivos, y se va produciendo una expansión, a lo que sigue posteriormente una contracción hacia la respuesta al problema, conformando los pasos una figura geométrica semejante a un rombo. Se basa en la aplicación interrelacionada de los métodos de análisis, síntesis, comparación, abstracción y generalización, partiendo de la descomposición del problema, hasta llegar a la integración necesaria cuando se elabora el informe final de la investigación. (Zayas, 2011, p. 68).

Gráfica 1. Rombo investigativo de la satisfacción laboral

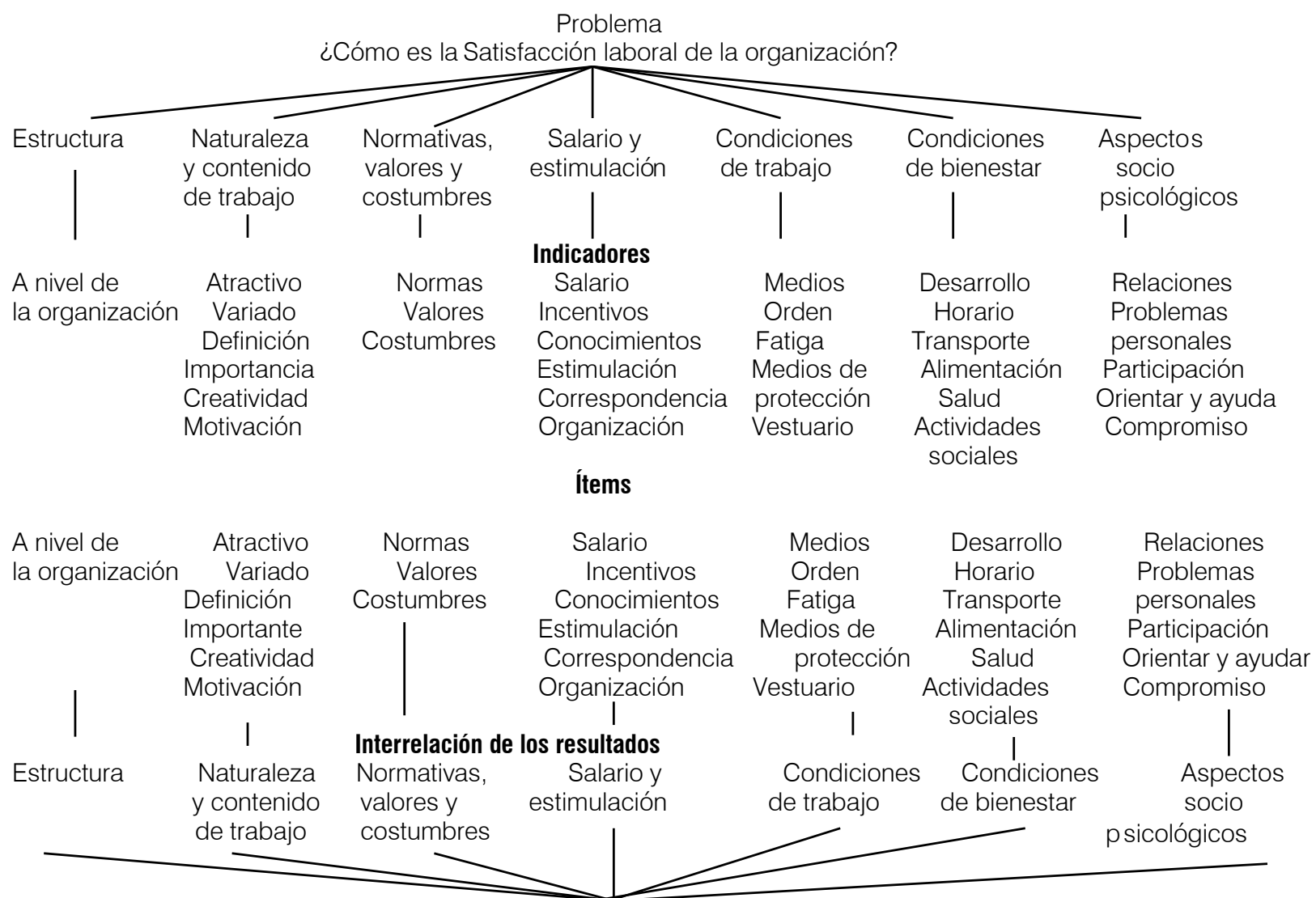

Respuesta

Fuente: Zayas et al. (2014) 
En el estudio de la validez de contenido, se les entrego al grupo expertos el modelo de la satisfacción laboral, para que lo evaluaran y aportaran su criterio con respecto a las dimensiones y a los indicadores. Después, a partir de los criterios del grupo evaluador, determinó cuáles eran los ítems necesarios para el desarrollo de la investigación, se presentó la escala que fue sometida a su consideración, para que valoraran si era necesario la inclusión o exclusión de algún elemento, revisaran la redacción de los ítems.

Para la validación de la escala se realizaron rondas con los expertos, donde se les facilitó la lista de preguntas contenidas en la escala, para buscar el consenso acerca de su aplicación en las áreas objeto de estudio. También se elaboró la guía para la realización de las entrevistas a los jefes de áreas y de las organizaciones sindicales.

\subsection{Validación del instrumento de medición la escala de satisfacción laboral}

El funcionamiento de la escala fue procesado mediante el paquete estadístico SPSS (versión 20.0) lo que permitió comprobar la consistencia interna y la fiabilidad de la escala, empleando para ello el coeficiente Alpha de Cronbach, obteniéndose un valor 0,873 siendo el índice de fiabilidad alto, lo que indica que el instrumento es fiable.

La validez del análisis factorial se comprobó a través del indice KMO (Kaiser-Meyer-Olkin) alcanzándose un valor 0,643 y una prueba de esfericidad de Bartlett con una Chi-cuadrado de 1743,063 para un $\alpha$ de 0,000 .

\subsubsection{Estudios para la obtención de evidencias externas de la validez del instrumento}

Para la obtención de las evidencias externas de la validez del instrumento, se evaluó a través de las entrevistas a los trabajadores y directivos, con la siguiente pregunta: ¿Cómo usted cree que es la satisfacción laboral de la organización?, y se correlacionaron los resultados que brindo la encuesta en excelente, bueno, regular, malo y muy mal, con los resultados de los índices individuales, y se obtuvo a través de coeficiente de Pearson un valor $0,712^{* * *}$ para un $\alpha$ de 0,01 .

\subsection{Instrumentos que se usaron en la investigación}

Los instrumentos que se usaron con el objetivo de conocer la satisfacción laboral de los trabajadores fueron una escala, reuniones de valoración y entrevistas.

La escala de satisfacción laboral está formada por 37 ítems, que recogen las 7 dimensiones esenciales, $y$ que indagan acerca de la satisfacción de cada persona, grupo y la organización. La escala fue construida bajo la técnica Likert, lo que le permite manifestar su "acuerdo" o "desacuerdo. La escala tuvo el objetivo de conocer el estado actual de los empleados sobre su satisfacción laboral.

Las preguntas están diseñadas para el procesamiento numérico por eso se responden en cinco categorías: Totalmente de acuerdo (5), De acuerdo (4), A veces (3), En desacuerdo (2) y Totalmente en desacuerdo (1) de forma tal que después de procesadas las escalas, posibilite al investigador o empresario tener una lectura por factor (dimensiones esenciales) $e$ indicadores. Los ítems 7, 9, 11, 12, 16 y 21 debe de invertirse a la hora de calificarse la escala.

Para la calificación y procesamiento de la información se confecciona una tabla y se procesa sobre la base de la media en sus diferentes formas, según la recopilación de la información, sea de un sujeto, el grupo, la organización y las dimensiones

Cada escala se procesa individualmente y se califica de: Excelente (con el rango de 5,00 hasta 4,00); Buena (con el rango de 3,99 hasta 3,50); Regular (con el rango de 3,49 hasta 3,00); Bajo (con el rango de 2,99 hasta 2,00) y Muy bajo (con el rango de 1,99 hasta 1,00 ) y así se evalúa también el grupo, la organización y las dimensiones.

Los resultados de la escala se combinaron con las entrevistas, para verificar posibles aspectos dudosos, interactuar con directivos, líderes sindicales y otros trabajadores. Las guías de las entrevistas fueron validadas por los expertos. 


\subsection{Procedimiento}

En la preparación general de la investigación, se seleccionó el grupo de los expertos y se realizó el involucramiento. El grupo de expertos estuvo encargado de explicar los objetivos, implicando a los demás miembros de la organización, a través de la divulgación de los objetivos principales y los beneficios de este estudio.

Para la realización de esta investigación se seleccionaron los instrumentos a aplicar, y se calculó la fiabilidad y la validez de los mismos, después se definió la forma en que se calificarían los instrumentos, y se estableció los formatos de las tablas.

En el proceso de selección de la muestra, era la intención de tratar de estudiar la población completa, pues una persona es importante y más si se habla de satisfacción laboral.

Se determinó cronograma de la aplicación y se divulgó en toda la organización. La aplicación de los instrumentos fue auto-administrada y se realizó en las áreas de la organización. La recolección de la información se realizó de forma organizada, debiendo quedar registrados y archivados los resultados de las escalas, reuniones y entrevistas, ya que son elementos informativos importantes para la valoración de la posible existencia de insatisfacción.

\subsection{Diseño experimental}

La variable dependiente es la satisfacción de trabajo y las variables independientes son: la estructura; la naturaleza y contenido de trabajo; normativas, valores y costumbres; el salario y la estimulación; las condiciones de trabajo; las condiciones de bienestar y aspectos socio-psicológicos (relaciones interpersonales-comunicación, liderazgo- toma de decisiones y el compromiso).

\subsection{Métodos y técnicas empleados en el procesa- miento de la información}

En el procesamiento de la información se utilizaron los métodos de expertos, método de trabajo en grupo (tormenta de ideas y dinámica de grupo), análi- sis de contenido y técnicas estadísticas como: Alfa de Cronbach, prueba de t, coeficiente de Pearson, coeficiente de concordancia de Kendall a través de SPSS 20.

\section{Resultados}

\subsection{Resultados del nivel individual}

El nivel individual expresa las características que diferencian a un individuo de los otros en la misma situación y realiza el análisis de los sujetos por cada uno de los rangos establecidos. Los resultados se muestran a continuación:

Del total de trabajadores, 4 obtienen resultados de Excelente en la satisfacción laboral, lo que representa 4,54\%; 34 trabajadores obtienen resultados de Bueno, lo que representa 38,6 \%; 43 obtienen resultados de Regular, lo que representa el 48,8 \%; 5 obtienen resultados de Bajo, lo que representa un $5,68 \%$ y 2 trabajadores obtuvieron resultado Muy bajo en la satisfacción laboral, representando el $2,27 \%$.

Cuadro 1. El rango de las categorías de la Satisfacción del trabajo se comporta así:

\begin{tabular}{|c|l|c|c|c|}
\hline & \multicolumn{3}{|c|}{ Satisfacción del trabajo } & \\
\hline No & \multicolumn{1}{|c|}{ Categoría } & Rango & Cantidad & $\%$ \\
\hline 1 & Excelente & $4,00-5,0$ & 4 & 4,54 \\
\hline 2 & Bueno & $3,99-3,50$ & 34 & 38,6 \\
\hline 3 & Regular & $3,49-3,00$ & 43 & 48,8 \\
\hline 4 & Bajo & $2,99-2,00$ & 5 & 5,68 \\
\hline 5 & Muy bajo & $1,99-1,0$ & 2 & 2,27 \\
\hline
\end{tabular}

Fuente: Cálculos propios

El 58,6\% de los resultados se encuentran por debajo del nivel deseado establecido de 3,50 que es de la categoría de Bueno, esto demuestra que existen problemas, los mismos que detallamos en los resultados por ítems, por lo que hay variables que están incidiendo desfavorablemente en la satisfacción laboral de los trabajadores. 
Tabla 1. Índice individual

\begin{tabular}{|l|c|c|c|c|c|c|c|c|c|c|c|c|c|c|c|c|c|c|}
\hline Índice & 1,89 & 1,97 & 2,65 & 2,70 & 2,78 & 2,92 & 2,95 & 3,00 & 3,08 & 3,11 & 3,14 & 3,19 & 3,22 & 3,24 & 3,30 & 3,32 & 3,35 & 3,38 \\
\hline Cantidad & 1 & 1 & 1 & 1 & 1 & 1 & 1 & 3 & 5 & 3 & 2 & 2 & 2 & 1 & 1 & 7 & 8 & 1 \\
\hline
\end{tabular}

\begin{tabular}{|l|c|c|c|c|c|c|c|c|c|c|c|c|c|c|c|c|c|c|c|c|}
\hline Índice & 3,41 & 3,43 & 3,49 & 3,51 & 3,57 & 3,59 & 3,62 & 3,65 & 3,70 & 3,73 & 3,76 & 3,78 & 3,81 & 3,84 & 3,89 & 3,95 & 3,97 & 4,00 & 4,08 & 4,27 \\
\hline Cantidad & 3 & 3 & 2 & 4 & 2 & 2 & 3 & 3 & 1 & 4 & 3 & 1 & 1 & 1 & 3 & 5 & 1 & 1 & 2 & 1 \\
\hline
\end{tabular}

Fuente: Cálculos propios.

\subsection{Resultados del nivel grupo}

Las áreas en que se realizo el estudio fueron los $\mathrm{Al}$ macenes y Protección física, que expresan las características grupales, que son distintas a los individuos de forma aislada.

El área de Almacenes se comportó de la siguiente forma:

Los ítems que mayor insatisfacción generan son: la proposición número 7 "Los medios materiales para realizar mi trabajo son insuficientes" con un índice de 2,97; la número 9 "Desarrollo mi trabajo en condiciones incómodas" con un índice de 2,9 y la proposición 12 "Las condiciones ambientales afectan mi salud" con 2,7 todas bajo la dimensión Condiciones de trabajo.

La proposición número 13 "El salario satisface mis necesidades básicas" con un índice de 1,88; la 14 "los incentivos que recibo satisfacen mis necesidades básicas" con un índice de 2,0; la proposición número 15 "El salario y los incentivos que recibo están acorde con el trabajo que realizo" con un índice de 2,3; la 16 "El sistema de estimulación establecido lo considero injusto" con un índice de 2,51; la proposición 17 "Existe una adecuada correspondencia entre los estímulos morales y materiales recibidos" con un índice de 2,6 las que se encuentran dentro de la dimensión salario y estimulación.

La proposición número 21 "Las normas y procedimientos en la organización son excesivas" con un índice de 2,5; incluida en la dimensión normas, valores y costumbres.

La proposición número 24 "Me siento satisfecho con las condiciones de desarrollo personal y profesional (capacitación, desarrollo, posibilidades de ascenso)" con un índice de 3,21 y la proposición número 26 "La empresa propicia buenas condiciones de transportación" con un índice de 2,77; las cuales forma parte de la dimensión condiciones de bienestar.

Los ítems que mayor satisfacción generan son: la proposición número 3 "Mi contenido de trabajo con su (objetivos, funciones, tareas), está bien definido" con un índice 4,03; la número 4 "Es importante el trabajo que realizo" 4,32; la 6 "Me gusta el trabajo que realizo" 4,00. En este caso se encuentran bajo la dimensión Naturaleza y contenido del trabajo.

La proposición número 8 "Mi área de trabajo permanece limpia y ordenada" con un índice de 3,96 y la número 10 "Existen en mi puesto de trabajo los medios de protección que yo necesito" 3,9; ambas pertenecientes a la dimensión condiciones de trabajo.

La proposición número 22 "Conozco los valores de mi organización" con un índice de 3,9 bajo la dimensión normas, valores y costumbres.

La proposición 30 "Las relaciones entre los trabajadores es buena" con un índice de 3,96 perteneciente a la dimensión aspectos socio-psicológicos. 
Tabla 2. Índices de los ítems en el grupo Almacenes

\begin{tabular}{|l|c|c|c|c|c|c|c|c|c|c|c|c|c|c|c|c|c|c|c|}
\hline Ítems & 1 & 2 & 3 & 4 & 5 & 6 & 7 & 8 & 9 & 10 & 11 & 12 & 13 & 14 & 15 & 16 & 17 & 18 & 19 \\
\hline Índice & 3,85 & 3,61 & 4,03 & 4,32 & 3,60 & 4,0 & 2,97 & 3,96 & 2,9 & 3,9 & 3,42 & 2,7 & 1,88 & 2,0 & 2,3 & 2,51 & 2,6 & 3,35 & 3,41 \\
\hline
\end{tabular}

\begin{tabular}{|l|c|c|c|c|c|c|c|c|c|c|c|c|c|c|c|c|c|c|c|}
\hline Ítems & 20 & 21 & 22 & 23 & 24 & 25 & 26 & 27 & 28 & 29 & 30 & 31 & 32 & 33 & 34 & 35 & 36 & 37 & Total \\
\hline Índice & 3,40 & 2,5 & 3,9 & 3,88 & 3,21 & 3,76 & 2,77 & 3,7 & 3,26 & 3,88 & 3,96 & 3,49 & 3,36 & 3,81 & 3,74 & 3,8 & 3,6 & 3,72 & 3,38 \\
\hline
\end{tabular}

Fuente: Cálculos propios.

Los ítems en el área Seguridad y Protección se comportaron de la siguiente forma:

Los ítems que mayor insatisfacción generan son: la proposición número 13 "El salario satisface mis necesidades básicas" con un índice de 2,5; la 14 "los incentivos que recibo satisfacen mis necesidades básicas" con un índice de 2,58; la proposición 17 "Existe una adecuada correspondencia entre los estímulos morales y materiales recibidos" con un índice de 3,2; y la proposición 19 "Cuando me destaco en el trabajo me reconocen ante el colectivo" con un índice de 3,0. Todas estas se encuentran dentro de la dimensión salario y estimulación.

La proposición número 7 "Los medios materiales para realizar mi trabajo son insuficientes" con un índice de 2,92; la número 9 "Desarrollo mi trabajo en condiciones incómodas" con un índice de 2,83; la número 11 "Tengo dificultades con el vestuario de trabajo" con un índice de 3,0 y la proposición 12 "Las condiciones ambientales afectan mi salud" con 3,17, que están bajo la dimensión condiciones de trabajo.

Los ítems que mayor satisfacción generan son: La proposición número 3 "Mi contenido de trabajo con sus (objetivos, funciones, tareas), está bien definido" con un índice 4,6; la número 4 "Es importante el trabajo que realizo" con un índice 4,75; la 6 "Me gusta el trabajo que realizo" con un índice 4,00. En este caso se encuentran bajo la dimensión naturaleza y contenido del trabajo.

La proposición número 8 "Mi área de trabajo permanece limpia y ordenada" con un índice de 4,25 perteneciente a la dimensión condiciones de trabajo.

La proposición número 22 "Conozco los valores de mi organización" con un índice de 4,00 bajo la dimensión normas, valores y costumbres.

La proposición 25 "Me siento cómodo con el horario de trabajo" con un índice de 4,08; la número 26 "La empresa propicia buenas condiciones de transportación" con un índice de 4,25; la número 27 "El servicio de alimentación es bueno" 4,5 bajo la dimensión condiciones de bienestar.

La proposición 30 "La relaciones entre los trabajadores es buena" con un índice de 4,08; la proposición 31 "Las relaciones entre los jefes y directivos de la empresa es buena" con un índice de 4,08; la proposición 32 "Las relaciones entre los jefes, directivos y trabajadores son buenas" con un índice de 4,08 y la proposición 33 "Mi jefe inmediato superior se preocupa por mis problemas personales" con un índice de 4,00 perteneciente a la dimensión aspectos socio-psicológicos. 
Tabla 3. Índices de los ítems en el grupo Protección física

\begin{tabular}{|l|c|c|c|c|c|c|c|c|c|c|c|c|c|c|c|c|c|c|c|}
\hline Ítem & 1 & 2 & 3 & 4 & 5 & 6 & 7 & 8 & 9 & 10 & 11 & 12 & 13 & 14 & 15 & 16 & 17 & 18 & 19 \\
\hline Índice & 3,66 & 3,41 & 4,58 & 4,75 & 3,66 & 40 & 2,91 & 4,25 & 2,83 & 3,58 & 3,0 & 3,16 & 2,5 & 2,58 & 3,33 & 3,66 & 3,16 & 3,66 & 3.0 \\
\hline
\end{tabular}

\begin{tabular}{|l|c|c|c|c|c|c|c|c|c|c|c|c|c|c|c|c|c|c|c|}
\hline Ítem & 20 & 21 & 22 & 23 & 24 & 25 & 26 & 27 & 28 & 29 & 30 & 31 & 32 & 33 & 34 & 35 & 36 & 37 & Total \\
\hline Índice & 3,91 & 3,66 & 4,0 & 3,83 & 3,41 & 4,08 & 4,25 & 4,5 & 3,83 & 3,83 & 4,08 & 4,08 & 4,08 & 4,0 & 3,66 & 3,91 & 4,16 & 4,0 & 3,70 \\
\hline
\end{tabular}

Fuente: Cálculos propios.

Los resultados de procesamiento de ítems de la satisfacción laboral entre el área de Almacenes y de Seguridad y protección en las pruebas no paramétricas, como es la prueba de los rangos con signo de Wilcoxon, se obtuvieron 27 pares rangos de empleados a favor de los que tienen la intención permanecer, 9 pares rangos de empleados que están a favor de los que tienen la intención abandono y 1 empate. En los estadísticos de contraste, se obtuvo una $\mathrm{Z}=$ $-4,022$ significativa para un $\alpha$ de 0.000 ).

\subsection{Dimensiones de la satisfacción laboral en el área de almacenes}

El comportamiento de las dimensiones de la satisfacción laboral en el área de almacenes se muestra a continuación ordenadas, de manera ascendente: Salario y estimulación con un índice de $(2,56)$; con- diciones de trabajo $(3,31)$; estructura $(3,4)$; normativas, valores y costumbres $(3,43)$; condiciones de bienestar $(3,47)$; aspectos socio- psicológicos $(3,61)$ y naturaleza y contenido de trabajo con un índice de $(3,89)$.

\subsection{Dimensiones de la satisfacción laboral en el área de seguridad y protección}

El comportamiento de las dimensiones de la satisfacción laboral en el departamento seguridad y protección se muestra a continuación ordenadas de manera ascendente: salario y estimulación revela un índice de $(3,02)$; condiciones de trabajo $(3,15)$; normativas, valores y costumbres $(3,83)$; condiciones de bienestar $(3,86)$; naturaleza y contenido de trabajo $(3,9)$, aspectos socio- psicológicos $(3,95)$ y estructura con un índice de $(4,0)$.

Tabla 4 Dimensiones de la satisfacción laboral por áreas

\begin{tabular}{|c|c|c|c|c|c|c|c|c|}
\hline No. & $\begin{array}{l}\text { Dimensiones } \\
\text { Áreas } \\
\text { de Trabajo }\end{array}$ & $\begin{array}{c}\text { Naturaleza y } \\
\text { contenido de } \\
\text { trabajo }\end{array}$ & $\begin{array}{c}\text { Condiciones } \\
\text { de trabajo }\end{array}$ & $\begin{array}{c}\text { Salario y } \\
\text { estimulación }\end{array}$ & $\begin{array}{l}\text { Condiciones } \\
\text { de bienestar }\end{array}$ & $\begin{array}{l}\text { Aspectos socio- } \\
\text { psicológicos }\end{array}$ & $\begin{array}{c}\text { Normativas, } \\
\text { valores y } \\
\text { costumbres }\end{array}$ & Estructura \\
\hline 1 & Almacén & 3,89 & 3,31 & 2,56 & 3,47 & 3,61 & 3,43 & 3,4 \\
\hline 2 & $\begin{array}{l}\text { Seguridad y } \\
\text { protección }\end{array}$ & 3,9 & 3,15 & 3,02 & 3,86 & 3,95 & 3,83 & 4,0 \\
\hline
\end{tabular}

Fuente: Cálculos propios.

Los resultados de procesamiento de las dimensiones de la satisfacción laboral entre el área de Almacenes y de Seguridad y protección, en las pruebas no paramétricas, como es la prueba de los rangos con signo de Wilcoxon, se obtuvieron 6 pares de rangos de empleados a favor de los que tienen la intención permanecer, 1 par de rangos de empleados que están a favor de los que tienen la intención abandono. En los estadísticos de contraste, se obtuvo una $\mathrm{Z}=$ $-2,028$ significativa para un $\alpha$ de 0,043. 


\subsection{Resultados de la organización}

El promedio del nivel de la satisfacción laboral se comportó con un índice de 3,42, evaluándose en la categoría de Regular.

Los ítems que mayor insatisfacción generan son: la proposición número 13 "El salario satisface mis necesidades básicas" con un índice de 2,0; la 14 "los incentivos que recibo satisfacen mis necesidades básicas" con un índice de 2,08; la proposición número 15 "El salario y los incentivos que recibo están acorde con el trabajo que realizo" con un índice de 2,44; la 16 "El sistema de estimulación establecido lo considero injusto" con un índice de 2,7; la proposición 17 "Existe una adecuada correspondencia entre los estímulos morales y materiales recibidos" con un índice de 2,64; la número 19 "Cuando me destaco en el trabajo se me reconoce en el colectivo" con un índice de 3,35. Todas estas se encuentran dentro de la dimensión salario y estimulación.

La proposición número 7 "Los medios materiales para realizar mi trabajo son insuficientes" con un índice de 3,0; la número 9 "Desarrollo mi trabajo en condiciones incómodas" con un índice de 2,9 y la proposición 12 "Las condiciones ambientales afectan mi salud" con 2,8 que están bajo la dimensión condiciones de trabajo.

La proposición número 21 "Las normas y procedimientos en la organización son excesivas" con un índice de 2,7; incluida en la dimensión normas, valores y costumbres.

La proposición número 24 "Me siento satisfecho con las condiciones de desarrollo personal y profesional (capacitación, desarrollo, posibilidades de ascenso)". con un índice de 3,2 y la proposición número 26 " $\mathrm{La}$ empresa propicia buenas condiciones de transportación" con un índice de 2,98; las cuales forman parte de la dimensión condiciones de bienestar.

Los ítems que mayor satisfacción generan son: la proposición número 1 "Mi trabajo me resulta atractivo" con un índice 3,83; la número 3 "Mi contenido de trabajo con sus (objetivos, funciones y tareas) están bien definido" $(4,10)$; la número 4 "Es importante el trabajo que realizo" (4,39); la 6 "Me gusta el trabajo que realizo" (4,6). En este caso se encuentran bajo la dimensión Naturaleza y contenido del trabajo.

La proposición número 8 "Mi área de trabajo permanece limpia y ordenada" con un índice de 4,00 y la número 10 "Existen en mi puesto de trabajo los medios de protección que yo necesito" $(3,86)$; ambas pertenecientes a la dimensión condiciones de trabajo.

La proposición número 22 "Conozco los valores de mi organización" con un índice de 3,95; la número 23 "Los valores de mi institución están acorde con mi persona" $(3,88)$; bajo la dimensión normas, valores y costumbres.

La proposición 25 "Me siento cómodo con el horario de trabajo" con un índice de 3,81; la número 27 "El servicio de alimentación es bueno" $(3,81)$; la 29 "Se realizan y se estimula la participación en actividades recreativas, culturales y deportivas" $(3,88)$; bajo la dimensión condiciones de bienestar.

La proposición 30 "La relaciones entre los trabajadores es buena" con un índice de 3,98 y la número 33 "Mi jefe inmediato superior se preocupa por mis problemas personales" $(3,84)$; pertenecientes a la dimensión aspectos socio-psicológicos.

Tabla 5. Promedio de los ítems de la escala

\begin{tabular}{|l|c|c|c|c|c|c|c|c|c|c|c|c|c|c|c|c|c|c|c|}
\hline Ítem & 1 & 2 & 3 & 4 & 5 & 6 & 7 & 8 & 9 & 10 & 11 & 12 & 13 & 14 & 15 & 16 & 17 & 18 & 19 \\
\hline Índice & 3,83 & 3,58 & 4,10 & 4,39 & 3,61 & 4,0 & 2,97 & 4,0 & 2,92 & 3,86 & 3,36 & 2,8 & 1,97 & 2,08 & 2,44 & 2,67 & 2,63 & 3,39 & 3,35 \\
\hline
\end{tabular}

\begin{tabular}{|l|c|c|c|c|c|c|c|c|c|c|c|c|c|c|c|c|c|c|c|}
\hline Ítem & 20 & 21 & 22 & 23 & 24 & 25 & 26 & 27 & 28 & 29 & 30 & 31 & 32 & 33 & 34 & 35 & 36 & 37 & Total \\
\hline Índice & 3,48 & 2,68 & 4,0 & 3,9 & 3,2 & 3,81 & 2,97 & 3,80 & 3,3 & 3,88 & 3,98 & 3,6 & 3,45 & 3,84 & 3,72 & 3,78 & 3,64 & 3,76 & 3,42 \\
\hline
\end{tabular}

Fuente: Cálculos propios. 


\subsection{Resultados por dimensiones}

Las dimensiones de la satisfacción del trabajo que se han afectado en orden ascendente son: salario y estimulación con un índice de $(2,56)$, con- diciones de trabajo $(3,29)$, normativas, valores y costumbres $(3,48)$, estructura $(3,5)$, condiciones de bienestar $(3,53)$, aspectos socio- psicológicos $(3,66)$ y naturaleza y contenido de trabajo con un índice de $(3,89)$.

Tabla 6. Dimensiones de la satisfacción laboral en la organización

\begin{tabular}{|l|c|c|c|c|c|c|c|c|}
\hline Dimensión & $\begin{array}{c}\text { Naturaleza y } \\
\text { contenido de } \\
\text { trabajo }\end{array}$ & $\begin{array}{c}\text { Condiciones } \\
\text { de trabajo }\end{array}$ & $\begin{array}{c}\text { Salario y } \\
\text { estimulación }\end{array}$ & $\begin{array}{c}\text { Condiciones } \\
\text { de bienestar }\end{array}$ & $\begin{array}{c}\text { Aspectos } \\
\text { socio- } \\
\text { psicológicos }\end{array}$ & $\begin{array}{c}\text { Normativas, } \\
\text { valores y } \\
\text { costumbres }\end{array}$ & Estructura & Total \\
\hline Índice & 3,9 & 3,29 & 2,6 & 3,53 & 3,66 & 3,48 & 3,5 & 3,42 \\
\hline
\end{tabular}

Fuente: Cálculos propios.

En el estudio, se determinó triangular las escalas, las entrevistas y las reuniones de grupo, para resumir los resultados, con las congruencias que tenían estas técnicas y la observación directa, donde se pudo apreciar con exactitud el comportamiento de los empleados.

Los análisis realizados permitieron determinar los problemas fundamentales que generan insatisfacción en los trabajadores de las áreas objeto de estudio. Como parte del estudio, y con el objetivo de lograr un nivel mayor de aproximación a los problemas y sus causas, se procedió a la realización de las reuniones con los líderes sindicales de la organización, pues los participantes coincidieron con los resultados obtenidos de las mismas y enriquecieron el estudio.

Los líderes sindicales plantearon los siguientes aspectos:

- Los trabajadores valoran insuficientes los estímulos materiales que reciben por su trabajo para satisfacer sus necesidades personales.

- No existe una adecuada correspondencia entre la estimulación moral y material y la actividad laboral que realizan.

- Se plantea que no se corresponde el salario con la actividad que realiza.

- El diseño de los puestos no se corresponde con las dimensiones antropométricas de los trabajadores, siendo incómodos para los mismos.
- Espacios de trabajo reducidos que dificultan la movilidad del personal y del montacargas por las áreas del almacén.

- Escases de mesas y sillas para desarrollar su actividad laboral con eficiencia.

- La iluminación de los puestos de trabajo no alcanza los niveles requeridos.

- Excesivo calor a causa de la falta de ventilación natural y artificial.

- Exposición a condiciones ambientales desfavorables.

- La mayoría de los trabajadores plantean no siempre propicia buenas condiciones de transportación.

- La mayor cantidad de trabajadores de las dos áreas objeto de estudio están de acuerdo que las normas y procedimientos en la organización son excesivos.

- Inconformidad con los métodos de dirección.

- Discrepancias con los jefes.

- Escasa posibilidad de superación.

- El trabajo realizan no está acorde con la especialidad que tienen. 
- Deficiente organización del trabajo.

- Problemas de vivienda.

- Carencia de círculos infantiles o seminternados.

Con el objetivo de lograr un nivel mayor de aproximación a los problemas y sus causas, se procedió a la realización de las entrevistas a los jefes de áreas y otros, donde se pudo comprobar la efectividad de la escala aplicada, pues los entrevistados coincidieron con los resultados obtenidos de las mismas y enriquecieron el estudio.

El análisis de las entrevistas realizadas, permitieron determinar y extraer las causas fundamentales que generan desmotivación en los trabajadores de las diferentes áreas. Las causas que expusieron los jefes de áreas que inhiben la satisfacción laboral en su organización son:

- Falta de estímulo material.

- En el trabajo existe mucha presión.

- Inconformidad con el salario.

- Mala remuneración.

- Lejanía del centro de trabajo.

- No le interesa el trabajo.

\section{Conclusiones}

Primeramente en este estudio se adoptó un concepto de satisfacción laboral, porque existen varias definiciones, es por ello epistemológicamente, teóricamente y metodológicamente, es necesario diferenciar, las actitudes de la satisfacción laboral, porque el concepto introduce cambios alrededor del objeto estudiado.

Los instrumentos en este estudio fueron probados, a través de su fiabilidad y su validez mediante el procesamiento estadístico, siendo ajustados en función de la organización.

Los conceptos teóricos, las dimensiones esenciales y variables son el enlace teoría-praxis. Es lo que va a materializar el objeto, en esta investigación son: la estructura; la naturaleza y contenido de trabajo; normativas, valores y costumbres; el salario y la estimulación; las condiciones de trabajo; las condiciones de bienestar y aspectos socio- psicológicos (Relaciones interpersonales y comunicación, liderazgo y toma de decisiones y el compromiso) todos los objetos, fenómenos, relaciones, tecnologías, las características del medio laboral que impregnen al sujeto y lo hagan significativo para ellos, por eso pensamos que la satisfacción laboral depende de la organización que se trate y del entorno socio-económico.

Los objetivos propuestos en estudio se cumplieron en toda su extensión, tanto como la capacidad para diagnosticar, evaluar, diferenciar, discriminar y explicar los factores que inciden en la satisfacción laboral.

Se pudo determinar un conjunto predominante de dimensiones e indicadores que identifican las principales causas, $e$ insuficiencias y condiciones existentes que limitan la satisfacción laboral.

La obtención de valores altos en una dimensión o en cada indicador, no significa que todo esté bien, o que este no deba ser tomado en consideración en las medidas acciones correctivas, porque estamos frente a un fenómeno dependiente de las condiciones objetivas y subjetivas y en última instancia depende del individuo y sus vivencias, valoraciones, percepciones, motivaciones y actitudes (Zayas et al., 2014).

En el plano grupal existen diferencias significativas entre el área Almacenes y el área Protección física a favor de esta última, porque tiene menos factores inhibidores de la satisfacción laboral, aunque las dimensiones del salario y estimulación y las condiciones de trabajo son similares.

En el plano organizacional general se manifiestan problemas que inhiben los niveles de satisfacción laboral relacionados con la dimensión salario y estimulación en todas las variables. En la dimensión condiciones de trabajo las condiciones técnicas organizativas existentes no se corresponden con el desarrollo de las actividades laborales, los medios materiales, las condiciones ergonómicas son insuficientes y la exposición a riesgos desfavorables para la salud humana. En la dimensión normas, valores y costumbres perciben que las normas y procedimientos en la organización son excesivas; las condiciones de transportación y las condiciones de desarrollo personal y profesional (capacitación, desarrollo, posibilidades 
de ascenso) son las dimensiones de las condiciones de bienestar que más impactan.

Estos son los resultados de la investigación se han detectado que se encuentran inhibiendo la satisfacción laboral, de los empleados, las áreas estudiadas y la organización, y a partir de los cuales se elaboraron las estrategias y acciones de mejora continua para el perfeccionamiento en la organización, permitiendo mejorar los niveles de satisfacción laboral y por ende satisfacer las expectativas de los trabajadores en una organización.

Así que se cumple con la hipótesis de investigación de que: Existen la mayoría de los trabajadores de la comercializadora mayorista al responder a la escala de la satisfacción laboral, valoran las dimensiones, indicadores y los ítems expresando con insatisfacciones en su trabajo.

\section{Estrategias y acciones}

Todos estos hallazgos de los instrumentos aplicados, con el método de la triangulación y a través del análisis de contenido, son factores inhibidores de la satisfacción laboral y nos llevaron al plan de estrategias y acciones.

\subsection{Perfeccionar el sistema de estimulación mo- ral y material en la organización}

\section{Acciones:}

- Evaluar y proponer modificaciones a las formas y sistemas de pago de forma tal que el pago del trabajo se realice de acuerdo al resultado individual

- Proponer un plan de atención a los empleados que incluya a los trabajadores más destacados.

\subsection{Mejorar las condiciones de trabajo}

Acciones:

- Elaborar un plan para el mejoramiento de las condiciones de trabajo y planificar los recursos financieros que lo respalden en dependencia de las posibilidades objetivas con que cuente la organización.
- Realizar estudios ergonómicos en los puestos de trabajos que resulten incómodos.

- Realizar un estudio en el sistema de seguridad y salud del trabajo, donde se evalué con profundidad los niveles de iluminación y ventilación existentes en las áreas, para determinar si existen problemas que estén afectando la salud de los empleados y de esta forma poder incidir sobre las causas.

- Valorar con la dirección de la aprobación de un presupuesto para ampliar el local del Almacén, con el objetivo de ubicar los productos de forma tal que no obstaculice el movimiento del personal y del montacargas.

- Evaluar y proponer a la Dirección para la compra del mobiliario que reúna los requisitos para el desarrollo del trabajo.

- Exámenes periódicos e investigaciones para detectar niveles de exposición a riesgos.

\subsection{Mejorar las condiciones de bienestar}

Acción:

- Garantizar los medios que aseguren la transportación de los obreros sin que esto compita con el proceso de distribución de productos.

\subsection{Perfeccionar las normativas, valores y cos- tumbres}

Acción:

- Revisión y perfeccionamiento de algunos procedimientos de trabajo, para evitar la tensión en el cumplimiento de determinadas funciones de trabajo.

\subsection{Mejorar aspectos socio- psicológicos}

Acción:

- Desarrollo de actividades para garantizar un ambiente agradable de trabajo, que permita una interacción positiva entre los directivos y trabajadores. 


\section{Referencias}

Álvarez, L. (2005). La satisfacción laboral su medición y evaluación; una experiencia cubana. [Consulta: febrero, 2012]. Disponible en: http://www.gestiopolis.com/Canales4/rrhh/ satislaboral.htm.

Brayfield, A., \&. Rothe, H. (1951) Journal of Appl. Pschology. Vol. 35, p. 307-11.En Abraham Kormak, (1978) "Psicología de la industria y de las organizaciones": Marova.

Cuesta, A. (1990). Organización del trabajo y psicología social. La Habana: Editorial Ciencias Sociales.

Davis, K, \& Newstrom, J. (1999). Comportamiento Humano en el trabajo: New York: Ed. McGraw-Hill.

Hackman, R. \& Oldham, G. (1980). Work redesign, USA: Ed. Addisson-Wesley.

Hackman, R. \& Lawler III, E. (1971). "Employee reactions to job characteristics". En: Journal of Psichology Monograph.

Hernández, R. et al. (2006). Metodología de la investigación 1. y 2 . México: Mc Graw - Hill.

Hoppock, R. (1935). Job Satisfaction. New York: Harper \& Row.

Herzberg, F., Mauser, B. \& Synderman, N. (1959). The motivation to work. Nueva York: Ed Wiles.

Larrainzar, M., Miñarro, J., Molinos, F. \& Verdú, M. (2001). "La satisfacción laboral en el hospital de Sant Cugat de Asepeyo". En: Capital humano, 143, p. 46-50.

Locke, E. (1976). The nature and causes of job satisfaction. Handbook of industry and organizational psychology. Chicago: Ed. Rand McNally College.

López, V. (1994). Las dimensiones esenciales de la motivación. En: Revista UPIICSA Tecnología, ciencia y cultura, Año II, I(3). Nueva época enero - abril. México.

Maslow A. (1954). Motivation and Personality. New York: Harper \& Row.

Mayo, E., (1933). The human problems of and industrial civilization, New York: Macmillan.

McGregor, D. (1960). The Human Side of Enterprise. Nueva York: McGraw-Hill.

Morse, N. (1953). Satisfaction in the white collar job. Michigan: University of Michigan.
Munsterberg, H. (1913). Psichology and industrial efficiency. En: Anastasi, A. (1979) Field of applied psychology $2^{\mathrm{a}}$ ed Nueva York: Mc Graw-Hill.

Padua, J. (1979). Técnicas de investigación aplicadas a las ciencias sociales. México, D. E: El Colegio de México/Fondo de Cultura Económica.

Peiró, J. (1991). Psicología de la Organización 2-1 y 2-2. Unidad didáctica IV. Universidad de Valencia.

Porter, L. \& Lawler, S. (1988). Teorías de Motivación. México: Edit. Prentice.

Robbins, S. (2000). "Comportamento Organizacional. Teoría y Práctica". Séptima. Méjico: Edición Prentice-Hall Hispanoamericana S.A.

Robbins, S. (2006). Comportamiento organizacional., México: Editorial Prentice Hall Hispanoamericana.

Robbins, S. \& Coulter, M. (1996). Administración. México: PrenticeHall Hispanoamericana, S.A.

Spector, P. (2002). Psicología Industrial y Organizacional: Investigación y Práctica. México: Manual Moderno.

Strauss, G. \& Sayles, L. (1992). Personal. México: EditorialPrentice Hall

Smith, P., Kendall, L. \& Hulin, C. (1969). The Measurement of Satisfaction in Work and Retirement: A strategy for the study of attitudes. Chicago: Rand McNally.

Taylor, F. (1911). The principles of Scientific Management. Nueva York: Ed. Harper y Bros.

Tubán, R. (2000). Medición del clima laboral en las organizaciones. Disponible en: http://www.tablero-decomando.com. [Consulta: abril, 2012].

Vroom, V. H. (1964). Work and Motivation, New York: Wiley.

Zayas, P. (1996). ¿Cómo seleccionar al personal de la empresa?, Ciudad Habana Ed. Academia.

Zayas, P. (2011). El Rombo Investigativo de las Ciencias Sociales: un método lógico-práctico en la concepción, proyección y ejecución de investigaciones. Disponible en: http://eumed.net/ libros/2010e/822/index.htm.

Zayas, P., Almaguer, M. \& Álvarez L. (2014). Instrumentos para el estudio y la evaluación de la satisfacción laboral en una organización, Revista Caribeña de las Ciencias Sociales, EUMED-NET. Disponible en: http://xn--caribea-9za.eumed. net/satisfacción-laboral/. 